\title{
Advanced small-cell colon carcinoma: a case report
}

\author{
Toshiaki Iwase ${ }^{1,2^{*}}$, Yoshinori Masuda', Takayuki Suzuki ${ }^{1}$, Osamu Takahashi ${ }^{1}$ and Masaru Miyazaki ${ }^{2}$
}

\begin{abstract}
Introduction: Small-cell colon carcinoma is a very rare disease among colon neoplasms; it is difficult to achieve long-term survival due to its aggressive tumor behavior. Here we report the long-term survival of a patient with advanced small-cell colon carcinoma achieved by a combination of surgery and continuous chemotherapy.

Case presentation: A 67-year-old Japanese man underwent abdominal computed tomography in our institution for follow up after gastrectomy, and abnormal thickness of the sigmoid colon wall was revealed. An endoscopy demonstrated a $20 \mathrm{~mm}$ Bormann 2 lesion with central ulceration located $20 \mathrm{~cm}$ from the anal verge. A sigmoidectomy was performed. Histologically, the tumor deeply invaded the tissue and extended beyond the serosa, and was diagnosed as small-cell carcinoma. Cisplatin plus irinotecan was administered for adjuvant chemotherapy. Nine months after surgery, a follow-up computed tomography showed an enlarged lymph node behind the inferior vena cava and a $15 \times 8 \mathrm{~mm}$ nodule located at the ventral side of the cecum. Under consideration of progressive disease, cisplatin plus irinotecan therapy was performed again using the same regimen. After nine cycles of cisplatin plus irinotecan therapy, a follow-up gastric endoscopy demonstrated external tumor invasion to the duodenum wall. Carboplatin plus etoposide therapy was selected as a third-line regimen. After six cycles of carboplatin plus etoposide therapy, the recurrence sites were maintained in a stable condition, and the survival time reached approximately 30 months after the initial surgery.

Conclusions: We report the long-term survival of a patient with advanced small-cell colon carcinoma. In the future, the accumulation and analysis of rare cases that obtain a better survival time will contribute to clarifying neuroendocrine carcinoma biology, and help to improve the prognosis.
\end{abstract}

Keywords: Chemotherapy, Small-cell carcinoma

\section{Introduction}

According to the classification of neuroendocrine tumors by the World Health Organization (WHO), smallcell colon carcinoma is categorized as a neuroendocrine carcinoma (NEC), which has high malignancy [1]. A NEC can occur anywhere in the gastrointestinal tract but it is rarely found in the colon: NEC has an incidence rate of only $0.6 \%$ of total colon malignancies [2]. Due to aggressive tumor behavior, distant metastases in internal organs or regional lymph nodes are often found at the same time as detection of a tumor. In addition to these facts, high recurrence rates contribute to poor survival,

\footnotetext{
* Correspondence: computerbody@hotmail.com

'Division of Surgery, Heiwa Hospital, 29-1 Higashiterao Nakadai Tsurumi-ku, Yokohana city, Kanagawa 230-0017, Japan

2Department of General Surgery, Chiba Graduate School of Medicine, 1-8-1 Inohana Chuo-ku, Chiba city, Chiba 260-8677, Japan
}

with a median survival of 10.5 months [2]. In patients with a metastatic lesion at the time of the surgery, the median survival time is only four months [3].

Here we report the long-term survival of a patient with advanced small-cell colon cancer who received continuous chemotherapy.

\section{Case presentation}

A 67-year-old Japanese man underwent abdominal computed tomography (CT) in our institution for follow up after gastrectomy; the CT revealed abnormal thickness of his sigmoid colon wall and further studies were planned. Two years previously, he had undergone a distal gastrectomy due to gastric cancer (moderately differentiated adenocarcinoma), and received S-1 (tegafurgimeracil, $100 \mathrm{mg}$ ) orally for 14 days followed by a sevenday rest period as adjuvant therapy for two years. He also

\section{Biomed Central}


had inactive hepatitis $C$. There were no abnormalities on physical examination except for an upper abdominal midline scar due to previous surgery, and his performance status was grade 0 . There were no abnormalities found on laboratory tests.

A double contrast radiological examination with barium enema showed a protruding lesion with a smooth surface at the distal end of the sigmoid colon, and the lumen was narrowed for approximately $2 \mathrm{~cm}$. An endoscopy demonstrated a $20 \mathrm{~mm}$ Bormann 2 lesion with central ulceration located $20 \mathrm{~cm}$ from the anal verge (Figure 1). No metastatic sites in other internal organs were confirmed by CT.

Multiple biopsies from the main site showed group $\mathrm{V}$ malignant cells that were positive for chromogranin A, synaptophysin, and CD56, and proved to be a NEC.

Under a diagnosis of NEC, a sigmoidectomy was performed. During the operation, three small hard white nodes were found at the mesenteric and pelvic cavity. The main tumor was located at $20 \mathrm{~cm}$ on the oral side of the peritoneal reflection, and dissected by three enlarged paracolic lymph nodes.

Macroscopically, a $15 \times 20 \mathrm{~mm}$ sub-mucosal tumor with the appearance of a small central ulceration was observed in the sigmoid colon (Figure 2).

Microscopically, the tumor deeply invaded the tissue and extended beyond the serosa. On hematoxylin and eosin staining, the tumor had high cellularity with appearance of hyperchromatic nuclei with irregular sheets, rosette arrangements, and alveolar structures (Figure 3). Immunohistochemical staining for chromogranin A, synaptophysin, and CD56 were also positive, which indicates an endocrine function (Figures 4 and 5). Several ductal structures were occupied by mucinous fluid and

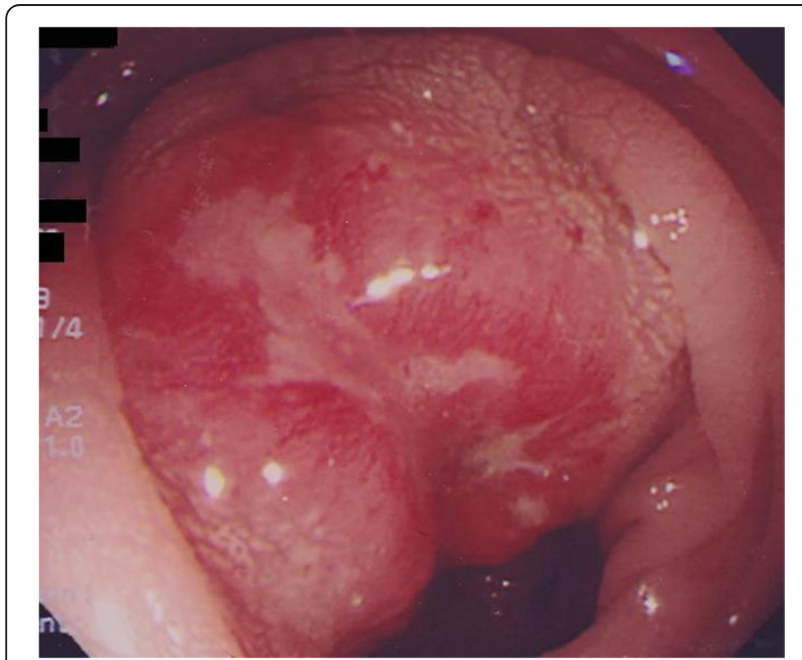

Figure 1 Endoscopic findings. An endoscopy demonstrated a $20 \mathrm{~mm}$ Bormann 2 lesion with a central ulceration located $20 \mathrm{~cm}$ from the anal verge.

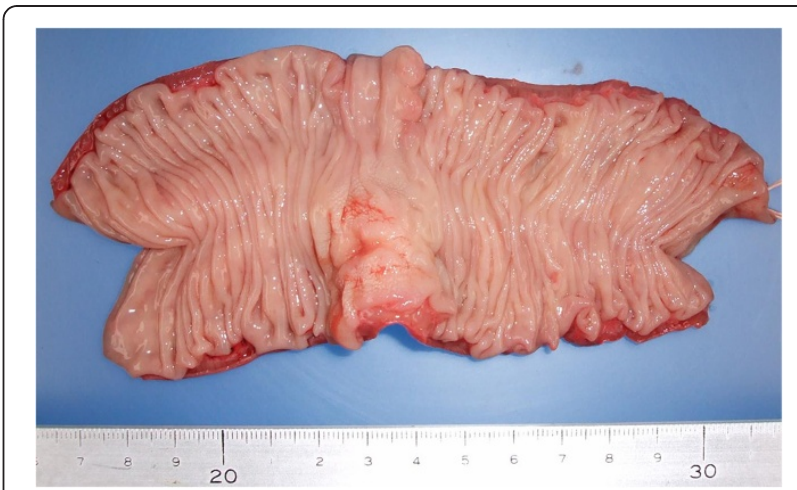

Figure 2 Macroscopic findings. A $15 \times 20 \mathrm{~mm}$ sub-mucosal tumor with the appearance of a small central ulceration was observed in the sigmoid colon.

positivity for periodic acid-Schiff (PAS), PAS-diastase (d-PAS), and Alcian blue was observed on the mucosal surface, suggesting the adenocarcinoma was included within the tumor. The nodules in the mesenteric and paracolic lymph nodes were also pathologically diagnosed as metastases. Finally, the tumor was diagnosed as a NEC (small-cell carcinoma).

Adjuvant chemotherapy was planned based on a regimen for small-cell lung carcinoma. Cisplatin plus irinotecan (cisplatin plus irinotecan therapy, cisplatin $60 \mathrm{mg} / \mathrm{m}^{2}$ day 1 and irinotecan $60 \mathrm{mg} / \mathrm{m}^{2}$ days 1,8 , and 15) were administered. Only a grade 2 side effect of transaminitis was observed during chemotherapy, and the patient recovered conservatively from this side effect. After finishing five cycles of chemotherapy, an evaluation CT showed no findings of recurrence. After cisplatin plus irinotecan therapy was finished, tegafur-uracil (UFT; 400mg) and leucovorin (UZEL, 75mg) were administered orally for 28 days

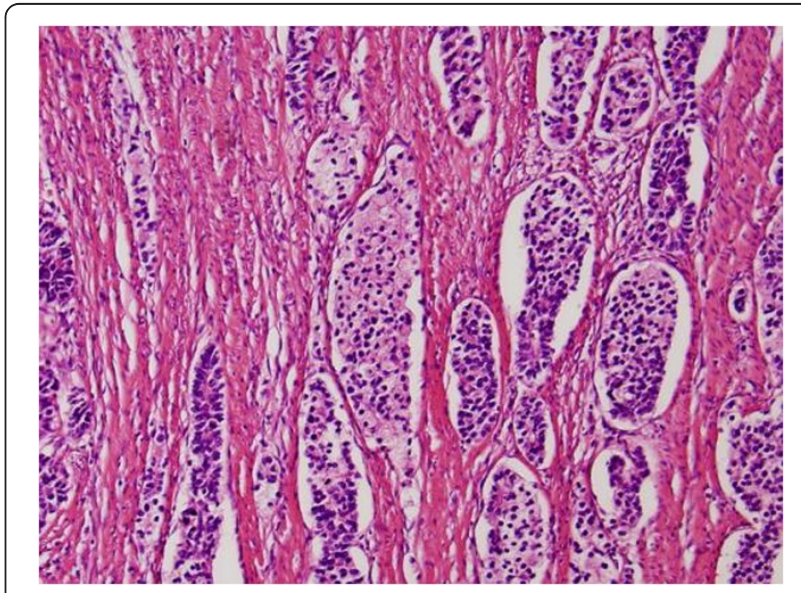

Figure 3 Pathological appearance of small-cell colon carcinoma on hematoxylin and eosin staining (original magnification $\times 100)$. 


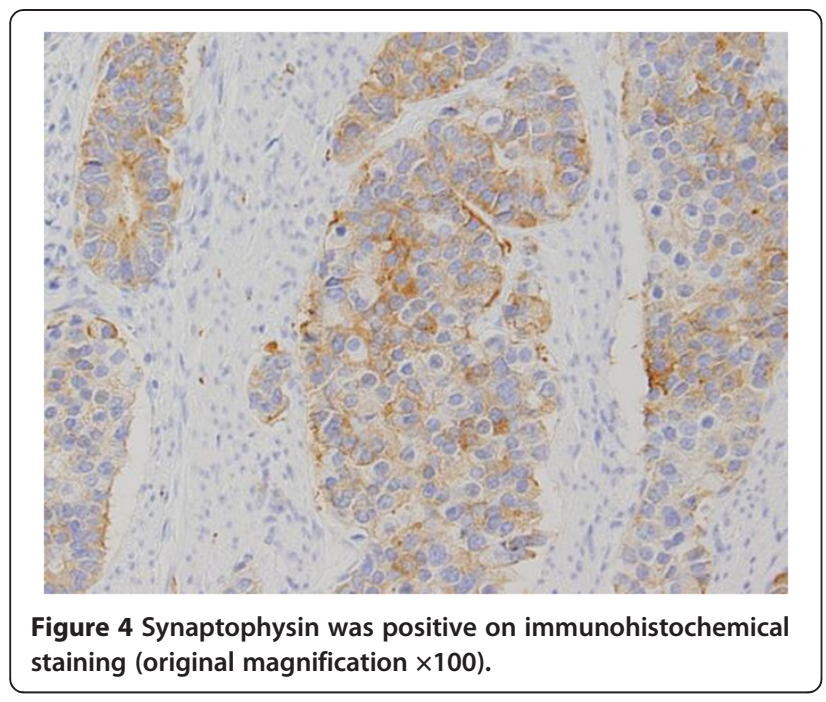

follow-up CT showed an enlarged lymph node behind the inferior vena cava and a $15 \times 8 \mathrm{~mm}$ nodule located at the ventral side of the cecum, which raised a suspicion of mesenteric dissemination. Under consideration of progressive disease, cisplatin plus irinotecan therapy was performed again using the same regimen. After nine cycles of cisplatin plus irinotecan therapy, a follow-up gastric endoscopy demonstrated external tumor invasion to the anterior duodenum wall due to growing peritoneal dissemination. It seemed that the tumor had acquired a resistance to cisplatin plus irinotecan therapy, and therefore carboplatin plus etoposide therapy, carboplatin plus etoposide therapy carboplatin 390mg day $1+$ etoposide $96 \mathrm{mg}, 80 \%$ dose, days 1, 2, 3 were selected as a third-line regimen. After six cycles of carboplatin plus etoposide therapy, the peritoneal dissemination sites had not expanded on CT findings, and the tumor invasion on the anterior duodenum wall had partially receded on endoscopic

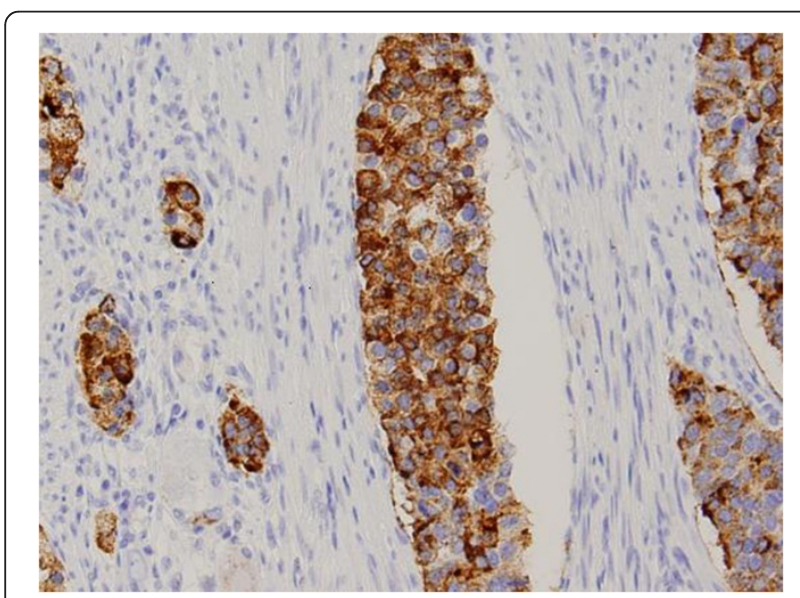

Figure 5 Chromogranin A was positive on immunohistochemical staining (original magnification $\times 100$ ). findings. At the present moment, the survival time has reached approximately 30 months after the initial surgery.

\section{Discussion}

According to the $2010 \mathrm{WHO}$ classification of neuroendocrine neoplasia (NET), NET was categorized into grade 1 and grade 2 based on the malignant potential of metastasis, and tumors with high malignancy were divided into NEC or mixed adenoendocrine carcinoma, which includes an element of adenocarcinoma of more than $30 \%$ [1]. The present case was pathologically diagnosed as a small-cell carcinoma mixed with poor to moderate adenocarcinoma elements of less than 30\%, and was categorized as NEC based on WHO classifications. When applying the present WHO classification, special attention should be paid because previously well or poorly differentiated endocrine carcinomas are redefined separately as NET grade 2 and NEC.

A definitive diagnosis was made histopathologically, and immunohistochemical staining greatly helps in diagnosis [4]. Saclarides et al. reported that more than a few cases initially diagnosed as carcinoid were changed to NEC after histopathological analysis [3]. In the present case, diagnosis was made preoperatively based on the immunohistochemical staining of chromogranin A, synaptophysin, and CD56. An endoscopic biopsy diagnosis is occasionally changed from well to moderately differentiated colon adenocarcinoma to NEC by pathological evaluations after surgery [3]. When any specific structures of NEC are histologically found, routine immunohistochemical staining contributes to appropriate diagnosis and continuous early treatment.

Chemotherapy for NEC is often performed based on the regimen for small-cell lung carcinoma. The latest guidelines for small-cell lung carcinoma from the National Comprehensive Cancer Network (NCCN) recommend etoposide plus platinum agents such as cisplatin or carboplatin for limited stage, and adding irinotecan instead of etoposide for extensive stage [5]. The present case initially received cisplatin plus irinotecan therapy, and secondly carboplatin plus etoposide therapy was selected because the tumor acquired resistance to cisplatin plus irinotecan therapy. In the present case, which was considered to have a high recurrence risk, UFT and UZEL were selected for maintenance and carboplatin plus etoposide therapy after the first cisplatin plus irinotecan therapy was administered based on a colon cancer regimen. Although the disease-free interval from the first cisplatin plus irinotecan therapy to recurrence was approximately four months, cisplatin plus irinotecan therapy was selected again. In the NCCN guidelines for small-cell lung cancer, when a recurrence is observed less than six months after finishing chemotherapy, a different treatment regimen is recommended [5]. It might have been better to change from cisplatin plus irinotecan 
therapy to carboplatin plus etoposide therapy at the time of recurrence because the tumor had already shown resistance to cisplatin plus irinotecan therapy.

It is very rare for patients with small-cell colon carcinoma to survive for approximately 30 months after being diagnosed with stage IV disease and peritoneal disseminations. Some reports have shown long-term survival despite an advanced stage by combination of operation and effective chemotherapy [6,7]. Unfortunately, patient characteristics associated with positive results from chemotherapy and favorable outcomes are still unknown because so few cases achieve long-term survival. A recent clinical trial showed the efficacy of amrubicin, an anthracycline agent with potent topoisomerase II inhibition activity, in improving survival time as second-line treatment for patients with small-cell lung cancer [8]. Although the number of cases was limited, Asayama et al. also reported the efficacy of amrubicin as first-line treatment for five cases of gastrointestinal endocrine carcinoma [9]. Earlier reports suggested that a certain percentage of patients with small-cell colon carcinoma had sensitivity for chemotherapy and achieved a better prognosis $[6,7]$.

In general, small-cell colon carcinoma has a high risk for recurrence and the follow-up method is important. Because recurrence sites are found in various organs, such as lymph nodes, liver, lung, and brain, less invasive whole body screening is needed. A recent study showed the effectiveness of somatostatin receptor scintigraphy on diagnosis and follow up by using an ${ }^{111}$ indium pentetreotide that specifically associated with the somatostatin receptor in NEC [10]. However, the superiority of $\mathrm{CT}$, magnetic resonance imaging, and somatostatin receptor scintigraphy is still controversial, and regular whole body screening and reassessment of chemotherapeutic effect is essential.

\section{Conclusion}

In this patient, long-term survival was achieved with a combination of surgery and chemotherapy, even though the cancer was at an advanced stage. The ideal strategy for advanced small-cell colon carcinoma has not yet been established. However, if it is determined before the operation that the tumor is advanced, then tumor resection should be performed, and selecting a chemotherapy regimen based on established guidelines will contribute to a better prognosis.

In the future, the accumulation and analysis of rare cases that obtain a better survival time will contribute to clarify NEC biology, and help to improve the prognosis.

\section{Consent}

Written informed consent was obtained from the patient for publication of this case report and accompanying images. A copy of the written consent is available for review by the Editor-in-Chief of this journal.

\section{Competing interests}

The authors declare that they have no competing interests.

\section{Authors' contributions}

TI: acquired the data and composed the manuscript. YM: interpretation and manuscript revision. TS: interpretation and manuscript revision. OT: interpretation and manuscript revision. MM: interpretation and manuscript revision. All authors read and approved the final manuscript.

Received: 25 October 2012 Accepted: 21 January 2013

Published: 18 March 2013

\section{References}

1. Rindi G, Arnold R, Bosman FT: Nomenclature and classification of neuroendocrine neoplasms of the digestive system. In WHO classification of tumours of the digestive system. 4th edition. Edited by Bosman FT, Carniero F, Hruban RH, Theise ND. Lyon, France: IARC; 2010:13-14.

2. Bernick PE, Klimstra DS, Shia J, Minsky B, Saltz L, Shi W, Thaler H, Guillem J, Paty $P$, Cohen AM, Wong WD: Neuroendocrine carcinomas of the colon and rectum. Dis Colon Rectum 2004, 47(2):163-169.

3. Saclarides TJ, Szeluga D, Staren ED: Neuroendocrine cancers of the colon and rectum. Results of a ten-year experience. Dis Colon Rectum 1994, 37(7):635-642

4. Ni SJ, Sheng WQ, Du X: Pathologic research update of colorectal neuroendocrine tumors. World J Gastroenterol 2010, 16(14):1713-1719.

5. Network NCC: NCCN Guidelines Version 2.2013 Small Cell Lung Cancer. http:// www.nccn.org/professionals/physician_gls/pdf/nscl.pdf.

6. Izuishi K, Arai T, Ochiai A, Ono M, Sugito M, Tajiri H, Saito N: Long-term survival in advanced small cell carcinoma of the colorectum: report of a case. Surg Today 2002, 32(1):72-74.

7. Power DG, Asmis TR, Tang LH, Brown K, Kemeny NE: High-grade neuroendocrine carcinoma of the colon, long-term survival in advanced disease. Med Oncol 2011, 28(Suppl 1):169-174.

8. Jotte R, Conkling P, Reynolds C, Galsky MD, Klein L, Fitzgibbons JF, McNally $\mathrm{R}$, Renschler MF, Oliver JW: Randomized phase II trial of single-agent amrubicin or topotecan as second-line treatment in patients with smallcell lung cancer sensitive to first-line platinum-based chemotherapy. J Clin Oncol 2011, 29(3):287-293.

9. Asayama M, Fuse N, Yoshino T, Yano T, Tahara M, Doi T, Fujii S, Ohtsu A: Amrubicin for the treatment of neuroendocrine carcinoma of the gastrointestinal tract: a retrospective analysis of five cases. Cancer Chemother Pharmacol 2011, 68(5):1325-1330.

10. Gotthardt M, Dirkmorfeld LM, Wied MU, Rinke A, Behe MP, Schlieck A, Hoffken H, Alfke H, Joseph K, Klose K, Behr TM, Arnold R: Influence of somatostatin receptor scintigraphy and CT/MRI on the clinical management of patients with gastrointestinal neuroendocrine tumors: an analysis in 188 patients. Digestion 2003, 68(2-3):80-85.

doi:10.1186/1752-1947-7-74

Cite this article as: Iwase et al: Advanced small-cell colon carcinoma: a case report. Journal of Medical Case Reports 2013 7:74.

\section{Submit your next manuscript to BioMed Central and take full advantage of:}

- Convenient online submission

- Thorough peer review

- No space constraints or color figure charges

- Immediate publication on acceptance

- Inclusion in PubMed, CAS, Scopus and Google Scholar

- Research which is freely available for redistribution 